\title{
Characterizations of groups generated by Kronecker sets
}

\author{
par ANDRÁS BIRÓ
}

\begin{abstract}
RÉsumÉ. Ces dernières années, depuis l'article [B-D-S], nous avons étudié la possibilité de caratériser les sous-groupes dénombrables du tore $T=\mathbf{R} / \mathbf{Z}$ par des sous-ensembles de $\mathbf{Z}$. Nous considérons ici de nouveaux types de sous-groupes: soit $K \subseteq T$ un ensemble de Kronecker (un ensemble compact sur lequel toute fonction continue $f: K \rightarrow T$ peut être approchée uniformément par des caractéres de $T$ ) et $G$ le groupe engendré par $K$. Nous prouvons (théorème 1) que $G$ peut être caractérisé par un sousensemble de $\mathbf{Z}^{2}$ (au lieu d'un sous-ensemble de $\mathbf{Z}$ ). Si $K$ est fini, le théorème 1 implique notre résultat antérieur de [B-S]. Nous montrons également (théorème 2) que si $K$ est dénombrable alors $G$ ne peut pas être caractérisé par un sous-ensemble de $\mathbf{Z}$ (ou une suite d'entiers) au sens de [B-D-S].
\end{abstract}

ABStRaCT. In recent years, starting with the paper [B-D-S], we have investigated the possibility of characterizing countable subgroups of the torus $T=\mathbf{R} / \mathbf{Z}$ by subsets of $\mathbf{Z}$. Here we consider new types of subgroups: let $K \subseteq T$ be a Kronecker set (a compact set on which every continuous function $f: K \rightarrow T$ can be uniformly approximated by characters of $T$ ), and $G$ the group generated by $K$. We prove (Theorem 1) that $G$ can be characterized by a subset of $\mathbf{Z}^{2}$ (instead of a subset of $\mathbf{Z}$ ). If $K$ is finite, Theorem 1 implies our earlier result in [B-S]. We also prove (Theorem 2 ) that if $K$ is uncountable, then $G$ cannot be characterized by a subset of $\mathbf{Z}$ (or an integer sequence) in the sense of [B-D-S].

\section{Introduction}

Let $T=\mathbf{R} / \mathbf{Z}$, where $\mathbf{R}$ denotes the additive group of the real numbers, $\mathbf{Z}$ is its subgroup consisting of the integers. If $x \in \mathbf{R}$, then $\|x\|$ denotes its distance to the nearest integer; this function is constant on cosets by $\mathbf{Z}$, so it is well-defined on $T$. A set $K \subseteq T$ is called a Kronecker set if it

Manuscrit reçu le 13 mai 2005.

Research partially supported by the Hungarian National Foundation for Scientific Research (OTKA) Grants No. T032236, T 042750 , T043623 and T049693 
is nonempty, compact, and for every continuous function $f: K \rightarrow T$ and $\delta>0$ there is an $n \in \mathbf{Z}$ such that

$$
\max _{\alpha \in K}\|f(\alpha)-n \alpha\|<\delta .
$$

If $K \subseteq T$ is a finite set, it is a Kronecker set if and only if its elements are independent over $\mathbf{Z}$ (this is essentially Kronecker's classical theorem on simultaneous diophantine approximation). There are many uncountable Kronecker sets, see e.g. [L-P], Ch. 1.

In [B-D-S] and in [B-S], we proved for a subgroup $G \subseteq T$ generated by a finite Kronecker set that $G$ can be characterized by a subset of the integers in certain ways. In fact we dealt with any countable subgroup of $T$ in [B-D$\mathrm{S}]$, and the result of [B-S] was generalized also for any countable subgroup in [B]. For further generalizations and strengthenings of these results, see [Bi1], [Bi2], [D-M-T], [D-K], [B-S-W].

In the present paper, we prove such a characterization of a group generated by a general Kronecker set by a subset of $\mathbf{Z}^{2}$ (instead of a subset of $\mathbf{Z}$ ). We also show, on the contrary, that using a subset of $\mathbf{Z}$, the characterization is impossible, if $K$ is uncountable. More precisely, we prove the following results.

Throughout the paper, let $K$ be a fixed Kronecker set, $G$ the subgroup of $T$ generated by $K$, and let $\epsilon>0$ be a fixed number. Write

$$
l(x)=\frac{-1}{\log _{2} x} \text { for } 0<x<1 / 2,
$$

and extend it to every $x \geq 0$ by $l(0)=0$, and $l(x)=1$ for $x \geq 1 / 2$.

Theorem 1. There is an infinite subset $A \subseteq \mathbf{Z}^{2}$ such that for every $\alpha \in G$ we have

$$
\sum_{\mathbf{n}=\left(n_{1}, n_{2}\right) \in A} l^{1+\epsilon}\left(\min \left(\left\|n_{1} \alpha\right\|,\left\|n_{2} \alpha\right\|\right)\right)<\infty,
$$

and if $\beta \in T$ satisfies

$$
\min \left(\left\|n_{1} \beta\right\|,\left\|n_{2} \beta\right\|\right)<\frac{1}{10}
$$

for all but finitely many $\mathbf{n}=\left(n_{1}, n_{2}\right) \in A$, then $\beta \in G$. Moreover, $A$ has the additional property that if $\alpha_{1}, \alpha_{2}, \ldots, \alpha_{t} \in G$ are finitely many given elements, then there is a function $f: A \rightarrow \mathbf{Z}$ such that $f(\mathbf{n})=n_{1}$ or $f(\mathbf{n})=n_{2}$ for every $\mathbf{n}=\left(n_{1}, n_{2}\right) \in A$, and for every $1 \leq i \leq t$ we have

$$
\sum_{\mathbf{n} \in A} l^{1+\epsilon}\left(\left\|f(\mathbf{n}) \alpha_{i}\right\|\right)<\infty .
$$

If $K$ is finite, the theorem of [B-S] follows at once from Theorem 1, since we can take all elements of $K$ as $\alpha_{1}, \alpha_{2}, \ldots, \alpha_{t}$ (see also Lemma 2 (i) in 
Section 3). Note that the statement of the Theorem in [B-S] contains a misprint: lim inf should be replaced by lim sup there.

Theorem 2. If $K$ is uncountable, and $A \subseteq \mathbf{Z}$ is an infinite subset, then

$$
G \neq\left\{\beta \in T: \lim _{n \in A}\|n \beta\|=0\right\} .
$$

This is in fact an easy corollary of a result of Aaronson and Nadkarni, but since the proof of that result is very sketchy in $[A-N]$, we present its proof (see Section 4, Prop. 1.).

We give the proof of Theorem 1 in Section 2. We mention that the basic idea is the same as in [Bi2]. Some lemmas needed in the proof of Theorem 1 are presented in Section 3. We remark that Lemma 4 is very important in the proof, and it provides the main reason why we need an $\epsilon>0$ in the theorem. The proof of Theorem 2 is given in Section 4. Section 5 contains a few comments and open questions.

\section{Proof of Theorem 1}

We will use Lemmas 2, 3 and 4, these lemmas are stated and proved in Section 3, so see that section if we refer to one of these lemmas.

If $x \in \mathbf{R}$, we also write $x$ for the coset of $x$ modulo $\mathbf{Z}$, so we consider $x$ as an element of $T$. The fractional part function $\{x\}$ is well-defined on $T$. Let $T^{(2)}$ be the subgroup of $T$ defined by

$$
T^{(2)}=\left\{\frac{a}{2^{N}}: N \geq 0,1 \leq a \leq 2^{N}\right\} .
$$

For $N \geq 0$ and $1 \leq a \leq 2^{N}$ let

$$
K_{N, a}=\left\{\alpha \in K: \frac{a-1}{2^{N}}<\{\alpha\}<\frac{a}{2^{N}}\right\} .
$$

Since $K$ is a Kronecker set, we can easily see that $K \cap T^{(2)}=\emptyset$, and so every $K_{N, a}$ is an open-closed subset of $K$, and

$$
K=\bigcup_{a=1}^{2^{N}} K_{N, a}
$$

(disjoint union). Let $F$ be the set of functions $f: K \rightarrow T^{(2)}$ which are constant on each small set of one of these subdivisions, i.e.

$$
F=\left\{f: K \rightarrow T^{(2)}:\left|f\left(K_{N, a}\right)\right| \leq 1 \quad \begin{array}{l}
\text { for some } N \geq 0 \\
\text { and for every } 1 \leq a \leq 2^{N}
\end{array}\right\},
$$

where $\left|f\left(K_{N, a}\right)\right|$ denotes the cardinality of the set $f\left(K_{N, a}\right)$, and we write $\leq 1$ because it may happen that some set $K_{N, a}$ is empty. Observe that $F$ is countable. Every element of $F$ is a continuous function on $K$, and 
$F$ is a group under pointwise addition. For a pair $(N, a)$ with $N \geq 0$ and $1 \leq a \leq 2^{N}$ let $F_{N, a} \leq F$ be the subgroup

$$
F_{N, a}=\left\{f \in F: f(\alpha)=0 \text { for } \alpha \in K \backslash K_{N, a},\left|f\left(K_{N, a}\right)\right| \leq 1\right\} .
$$

For any $N \geq 0$ let $g_{N} \in F$ be defined by

$$
g_{N}(\alpha)=\frac{a}{2^{N}} \text { for every } \alpha \in K_{N, a} \text { and for every } 1 \leq a \leq 2^{N},
$$

and let $f_{N, a, r} \in F_{N, a}$ be defined by $\left(N \geq 0,1 \leq a \leq 2^{N}, r \geq 1\right.$ are fixed):

$$
f_{N, a, r}(\alpha)= \begin{cases}2^{-r}, & \text { if } \alpha \in K_{N, a} \\ 0, & \text { if } \alpha \in K \backslash K_{N, a} .\end{cases}
$$

Clearly

$$
\max _{\alpha \in K}\left\|g_{N}(\alpha)-\alpha\right\| \leq 2^{-N} \text { for every } N \geq 0 .
$$

Remark that the functions $g_{N}$ are not necessarily distinct, but if $N \geq 0$ is fixed, then

$$
\left|\left\{\nu \geq 0: g_{\nu}=g_{N}\right\}\right|<\infty,
$$

since otherwise (2.1), applied for the elements $\nu$ of this set, would give $g_{N}(\alpha)=\alpha$ for every $\alpha \in K$, which is impossible by $K \cap T^{(2)}=\emptyset$.

For every $f \in F$ take a number $C(f)>0$, and for every $N \geq 0$ a number $R(N)>0$, we assume the following inequalities:

$$
\sum_{f \in F} C(f)^{-\epsilon}<\infty, \quad \sum_{N=0}^{\infty} R(N)^{-\epsilon}<\infty,
$$

and (it is possible by (2.2)):

$$
C\left(g_{N}\right)>N \text { for every } N \geq 0 .
$$

For every $f \in F$ and for every integer $j \geq 1$ we take an integer $m_{j}(f)$ such that

$$
\max _{\alpha \in K}\left\|f(\alpha)-m_{j}(f) \alpha\right\|<2^{-j-2^{j} C(f)}
$$

which is possible, since $K$ is a Kronecker set. Moreover, we can assume that if $j, j^{\star} \geq 1, f, f^{\star} \in F$, then

$$
m_{j^{\star}}\left(f^{\star}\right) \neq m_{j}(f) \text { if }(j, f) \neq\left(j^{\star}, f^{\star}\right) .
$$

Indeed, there are countably many pairs $(j, f)$, and for a fixed pair $(j, f)$ there are infinitely many possibilities for $m_{j}(f)$ in $(2.5)$, so we can define recursively the integers $m_{j}(f)$ to satisfy $(2.5)$ and $(2.6)$.

Let $j(N, a, r) \geq 1$ be integers for every triple $(N, a, r) \in V$, where

$$
V=\left\{(N, a, r): N \geq 0,1 \leq a \leq 2^{N}, r>R(N)\right\},
$$


satisfying that if $\left(N^{\star}, a^{\star}, r^{\star}\right) \in V$ is another such triple, then

$$
j(N, a, r) \neq j\left(N^{\star}, a^{\star}, r^{\star}\right) \text {, if }(N, a, r) \neq\left(N^{\star}, a^{\star}, r^{\star}\right) .
$$

We easily see from (2.6) and (2.7) that for $(N, a, r),\left(N^{\star}, a^{\star}, r^{\star}\right) \in V$ we have

$$
m_{j(N, a . r)}\left(f_{N, a, r}\right) \neq m_{j\left(N^{\star}, a^{\star}, r^{\star}\right)}\left(f_{N^{\star}, a^{\star}, r^{\star}}\right) \text {, if }(N, a, r) \neq\left(N^{\star}, a^{\star}, r^{\star}\right) .
$$

Define

We claim that

$$
H_{1}=\left\{m_{j(N, a, r)}\left(f_{N, a, r}\right):(N, a, r) \in V\right\} .
$$

$$
\sum_{n \in H_{1}} l^{1+\epsilon}(\|n \alpha\|)<\infty
$$

for every $\alpha \in K$. Indeed, let $\alpha \in K$ be fixed. We have

$$
\left\|m_{j(N, a, r)}\left(f_{N, a, r}\right) \alpha\right\| \leq\left\|f_{N, a, r}(\alpha)\right\|+2^{-1-2^{j(N, a, r)} C\left(f_{N, a, r}\right)}
$$

by (2.5). Now, on the one hand,

$$
\sum_{a=1}^{2^{N}} l^{1+\epsilon}\left(\left\|f_{N, a, r}(\alpha)\right\|\right)=l^{1+\epsilon}\left(2^{-r}\right), \quad \sum_{N=0}^{\infty} \sum_{r>R(N)} l^{1+\epsilon}\left(2^{-r}\right)<\infty
$$

by (2.3); on the other hand, using (2.7) and (2.3), we get

$$
\sum_{(N, a, r) \in V} l^{1+\epsilon}\left(2^{-1-2^{j(N, a, r)} C\left(f_{N, a, r}\right)}\right) \leq \sum_{f \in F} \sum_{j \geq 1}\left(C(f) 2^{j}\right)^{-(1+\epsilon)}<\infty .
$$

In view of Lemma 2 (i), (2.11)-(2.13), and the definition of $H_{1}$ in (2.9), we get (2.10).

If $s$ is a nonnegative integer, the following set is a compact subset of $T$ :

$$
K_{s}=\left\{\begin{array}{l}
\alpha=\sum_{i=1}^{t} k_{i} \alpha_{i}: \begin{array}{l}
t \geq 1, \alpha_{1}, \alpha_{2}, \ldots, \alpha_{t} \in K \\
k_{1}, k_{2}, \ldots, k_{t} \in \mathbf{Z}, \sum_{i=1}^{t}\left|k_{i}\right| \leq s
\end{array}
\end{array}\right\} .
$$

Lemma 1. There is a subset $H$ of the integers such that $H_{1} \subseteq H$ and on the one hand we have

$$
\sum_{n \in H} l^{1+\epsilon}(\|n \alpha\|)<\infty
$$

for every $\alpha \in K$; on the other hand, if $\beta \in T$ has the property that

$$
\|n \beta\|<\frac{1}{10}
$$

for all but finitely many $n \in H$, then there is a group homomorphism $\phi_{\beta}=\phi: F \rightarrow T$ which satisfies the following properties:

(i) for all but finitely many pairs $(f, j)$ with $f \in F, j \geq 1$ we have

$$
\left\|\phi(f)-m_{j}(f) \beta\right\|<2^{-C(f)-j} ;
$$


(ii) for every $(N, a)$ pair with $N \geq 0,1 \leq a \leq 2^{N}$, if $K_{N, a} \neq \emptyset$, there is a unique integer $k_{N, a}$ for which

$$
\phi(f)=k_{N, a} f(\alpha)
$$

for every $f \in F_{N, a}$, where $\alpha \in K_{N, a}$ is arbitrary; if $K_{N, a}=\emptyset$, we put $k_{N, a}=0$, and then for large $N$ we have

$$
\max _{1 \leq a \leq 2^{N}}\left|k_{N, a}\right| \leq 2^{R(N)}
$$

(iii) if $N$ is large enough, then writing $s=\sum_{a=1}^{2^{N}}\left|k_{N, a}\right|$, there is an $\alpha \in K_{s}$ such that

$$
\|\alpha-\beta\| \leq \frac{1}{N}+s 2^{-N}
$$

Proof. Define

$$
H_{2}=\left\{2^{r}\left(m_{j+1}(f)-m_{j}(f)\right): f \in F, j \geq 1,0 \leq r \leq j-1+C(f)\right\} .
$$

Let us choose for every triple $f_{1}, f_{2}, f_{3} \in F$ with $f_{3}=f_{1}+f_{2}$ an infinite subset $J_{f_{1}, f_{2}, f_{3}}$ of the positive integers such that (the first summation below is over every such triple from $F$ )

$$
\Sigma:=\sum_{f_{3}=f_{1}+f_{2}} \sum_{j \in J_{f_{1}, f_{2}, f_{3}}}\left(2^{j} \min \left(C\left(f_{1}\right), C\left(f_{2}\right), C\left(f_{3}\right)\right)\right)^{-\epsilon}<\infty .
$$

Since $C(f)>0$ for every $f \in F, \epsilon>0$ and $F$ is countable, this is obviously possible. Then define (we mean again that $f_{1}, f_{2}, f_{3}$ run over every such triple from $F$ )

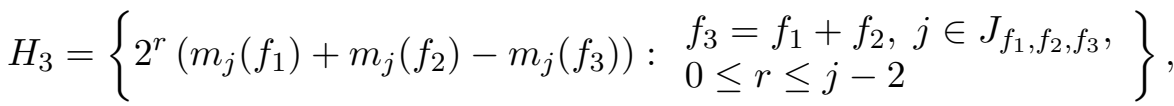

$$
\begin{aligned}
& H_{4}=\left\{2^{r}\left(m_{1}\left(g_{N}\right)-1\right): N \geq 1,0 \leq r \leq \log _{2} N\right\} .
\end{aligned}
$$

Let $H=\bigcup_{i=1}^{4} H_{i}$. We first prove (2.14). If $f \in F, j \geq 1$ and $\alpha \in K$, then

$$
\left\|\left(m_{j+1}(f)-m_{j}(f)\right) \alpha\right\| \leq 2^{-(j+C(f)-1)-\left(2^{j}-1\right) C(f)}
$$

by (2.5), therefore, using also Lemma 2 (ii) and (2.3), we obtain

$$
\sum_{n \in H_{2}} \max _{\alpha \in K} l^{1+\epsilon}(\|n \alpha\|) \leq m \sum_{f \in F} \sum_{j \geq 1} C(f)^{-\epsilon}\left(2^{j}-1\right)^{-\epsilon}<\infty .
$$

If $\alpha \in K, f_{1}, f_{2}, f_{3} \in F, f_{3}=f_{1}+f_{2}$ and $j \in J_{f_{1}, f_{2}, f_{3}}$, then by (2.5) we get

$$
\left\|\left(m_{j}\left(f_{1}\right)+m_{j}\left(f_{2}\right)-m_{j}\left(f_{3}\right)\right) \alpha\right\| \leq 2^{-(j-2)} 2^{-2^{j} \min \left(C\left(f_{1}\right), C\left(f_{2}\right), C\left(f_{3}\right)\right)},
$$

and so by Lemma 2 (ii) and (2.20) we get

$$
\sum_{n \in H_{3}} \max _{\alpha \in K} l^{1+\epsilon}(\|n \alpha\|) \leq m \Sigma<\infty .
$$


If $N \geq 1$ and $\alpha \in K$, then

$$
\left\|\left(m_{1}\left(g_{N}\right)-1\right) \alpha\right\| \leq\left\|m_{1}\left(g_{N}\right) \alpha-g_{N}(\alpha)\right\|+\left\|g_{N}(\alpha)-\alpha\right\| \leq 2^{1-N}
$$

by (2.1), (2.4) and (2.5), so by the definition of $H_{4}$, we obtain

$$
\sum_{n \in H_{4}} \max _{\alpha \in K} l^{1+\epsilon}(\|n \alpha\|) \leq \sum_{N=1}^{\infty}\left(1+\log _{2} N\right) l^{1+\epsilon}\left(2^{1-N-\log _{2} N}\right)<\infty .
$$

The relations (2.10), (2.22), (2.24) and (2.26) prove (2.14).

Now, assume that for a $\beta \in T$ we have an $n_{0}>0$ such that (2.15) is true if $n \in H$ and $|n|>n_{0}$. Since $K$ is a Kronecker set, so $\|n \alpha\|>0$ for $0 \neq n \in \mathbf{Z}, \alpha \in K$. Therefore, we see from (2.21) (and (2.3)) that

$$
0<\left|m_{j+1}(f)-m_{j}(f)\right| \leq n_{0}
$$

can hold only for finitely many pairs $f \in F, j \geq 1$; we see from (2.23) that if $f_{1}, f_{2}, f_{3} \in F$ are given with $f_{3}=f_{1}+f_{2}$, then

$$
0<\left|m_{j}\left(f_{1}\right)+m_{j}\left(f_{2}\right)-m_{j}\left(f_{3}\right)\right| \leq n_{0}
$$

can hold only for finitely many $j \geq 1$; and from (2.25) that

$$
0<\left|m_{1}\left(g_{N}\right)-1\right| \leq n_{0}
$$

can hold only for finitely many $N$. Then, by Lemma 3, we obtain the following inequalities (using $H_{2} \subseteq H, H_{3} \subseteq H, H_{4} \subseteq H$, respectively):

$$
\left\|\left(m_{j+1}(f)-m_{j}(f)\right) \beta\right\|<\frac{1 / 10}{2^{j-2+C(f)}}
$$

for all but finitely many pairs $f \in F, j \geq 1$;

$$
\left\|\left(m_{j}\left(f_{1}\right)+m_{j}\left(f_{2}\right)-m_{j}\left(f_{3}\right)\right) \beta\right\|<\frac{1 / 10}{2^{j-2}}
$$

for every triple $f_{1}, f_{2}, f_{3} \in F$ with $f_{3}=f_{1}+f_{2}$ and for large enough $j \in J_{f_{1}, f_{2}, f_{3}}$

$$
\left\|\left(m_{1}\left(g_{N}\right)-1\right) \beta\right\|<\frac{1 / 10}{N / 2}
$$

for large enough $N$.

Then from (2.27), for all but finitely many pairs $f \in F, j_{1} \geq 1$ we have

$$
\left\|\left(m_{j_{2}}(f)-m_{j_{1}}(f)\right) \beta\right\|<\frac{2 / 5}{2^{C(f)}} \sum_{j=j_{1}}^{j_{2}-1} 2^{-j}
$$

for every $j_{2}>j_{1}$. This implies that $m_{j}(f) \beta$ is a Cauchy sequence for every $f \in F$, so

$$
\phi(f):=\lim _{j \rightarrow \infty} m_{j}(f) \beta
$$

exists, (2.16) is satisfied for all but finitely many pairs $f \in F, j \geq 1$ by (2.30), and since every $J_{f_{1}, f_{2}, f_{3}}$ is an infinite set, $\phi: F \rightarrow T$ is a group 
homomorphism by (2.28) and (2.31). We also see that for large $N$, by (2.16), (2.4) and (2.29), we have

$$
\left\|\phi\left(g_{N}\right)-\beta\right\| \leq \frac{1}{N}
$$

If $(N, a)$ is a fixed pair with $N \geq 0,1 \leq a \leq 2^{N}$ and $K_{N, a} \neq \emptyset$, then

$$
\left\|\phi\left(f_{N, a, r}\right)\right\| \leq\left\|\phi\left(f_{N, a, r}\right)-m_{j(N, a, r)}\left(f_{N, a, r}\right) \beta\right\|+\left\|m_{j(N, a, r)}\left(f_{N, a, r}\right) \beta\right\|,
$$

and so

$$
\limsup _{r \rightarrow \infty}\left\|\phi\left(f_{N, a, r}\right)\right\| \leq \frac{1}{10}
$$

by (2.16), (2.7), using also the assumption on $\beta,(2.8)$ and $H_{1} \subseteq H$. Then (2.17) follows from Lemma 4 , because $F_{N, a}$ is obviously isomorphic to $T^{(2)}$. We now prove (2.18). Assume that $N$ is large and

$$
\left|k_{N, a}\right|>2^{R(N)}
$$

for some $1 \leq a \leq 2^{N}$. Take an integer $r$ such that

$$
2\left|k_{N, a}\right| \leq 2^{r} \leq 4\left|k_{N, a}\right| \text {. }
$$

Then $r>R(N)$, so $m_{j(N, a, r)}\left(f_{N, a, r}\right) \in H_{1} \subseteq H$, and so for large $N$ we have (see $(2.8)$ ) that

$$
\left\|m_{j(N, a, r)}\left(f_{N, a, r}\right) \beta\right\|<\frac{1}{10} .
$$

But (2.34) and (2.17) imply

$$
\left\|\phi\left(f_{N, a, r}\right)\right\| \geq \frac{1}{4}
$$

which contradicts (2.35) for large $N$ by (2.16) and (2.7). Therefore (2.33) cannot be true for large $N$, so (2.18) is proved. To prove (2.19), if $N \geq 0$, $1 \leq a \leq 2^{N}$ are arbitrary and $k_{N, a} \neq 0$, which implies $K_{N, a} \neq \emptyset$ by definition, we take an $\alpha_{N, a} \in K_{N, a}$, and then, by the definition of $g_{N}$ and by the already proved properties of $\phi$, we have

$$
\left\|\phi\left(g_{N}\right)-\sum_{1 \leq a \leq 2^{N}, k_{N, a} \neq 0} k_{N, a} \alpha_{N, a}\right\| \leq 2^{-N} \sum_{a=1}^{2^{N}}\left|k_{N, a}\right|,
$$

and together with (2.32), this proves (2.19).

Proof of Theorem 1. For every $N \geq 0$ we take some integer $j(N) \geq 1$ such that the sequence $j(N)$ is strictly increasing and

$$
\sum_{N=0}^{\infty} 2^{N-1}(R(N)+2)^{2} l^{1+\epsilon}\left(2^{-j(N)}\right)<\infty .
$$

Let

$$
U=\left\{(N, a): N \geq 0,1 \leq a \leq 2^{N-1}, K_{N, 2 a-1} \neq \emptyset, K_{N, 2 a} \neq \emptyset\right\},
$$


define $A^{\star} \subseteq \mathbf{Z}^{2}$ as

$$
A^{\star}=\left\{\left(m_{j(N)}\left(f_{N, 2 a-1, r_{1}}\right), m_{j(N)}\left(f_{N, 2 a, r_{2}}\right)\right): \begin{array}{l}
(N, a) \in U, \\
1 \leq r_{1}, r_{2} \leq R(N)+2
\end{array}\right\},
$$

and let $A=A^{\star} \cup\{(n, n): n \in H\}$. Note that if $(N, a),\left(N^{\star}, a^{\star}\right) \in U$, and $1 \leq r_{1} \leq R(N)+2,1 \leq r_{1}^{\star} \leq R\left(N^{\star}\right)+2$, then

$$
m_{j(N)}\left(f_{N, 2 a-1, r_{1}}\right) \neq m_{j\left(N^{\star}\right)}\left(f_{N^{\star}, 2 a^{\star}-1, r_{1}^{\star}}\right) \text {, if }(N, a) \neq\left(N^{\star}, a^{\star}\right) \text {. }
$$

Indeed, this follows from the fact that $j$ is strictly increasing (so one-toone), using (2.6) and the definition of $U$.

Assume that $\beta \in T$ satisfies (1.2) for all but finitely many $\mathbf{n}=\left(n_{1}, n_{2}\right) \in$ $A$. Then (2.15) is true for all but finitely many $n \in H$, we can apply Lemma 1. If $N$ is large, and we assume that $k_{N, 2 a-1} \neq 0$ and $k_{N, 2 a} \neq 0$ for some $1 \leq a \leq 2^{N-1}$ (this implies $(N, a) \in U$ by the definitions), then by (2.18) we can take a pair $1 \leq r_{1}, r_{2} \leq R(N)+2$ such that

$$
2\left|k_{N, 2 a-1}\right| \leq 2^{r_{1}} \leq 4\left|k_{N, 2 a-1}\right|, \quad 2\left|k_{N, 2 a}\right| \leq 2^{r_{2}} \leq 4\left|k_{N, 2 a}\right| .
$$

Then by (2.17), we have

$$
\left\|\phi\left(f_{N, 2 a-1, r_{1}}\right)\right\| \geq \frac{1}{4}, \quad\left\|\phi\left(f_{N, 2 a, r_{2}}\right)\right\| \geq \frac{1}{4}
$$

and, in view of (2.16), $j(N) \rightarrow \infty$, the definition of $A,(2.37)$ and the property of $\beta$, this is a contradiction for large $N$. Therefore, if $N$ is large, then $k_{N, 2 a-1} k_{N, 2 a}=0$ for every $1 \leq a \leq 2^{N-1}$, and since clearly $k_{N, 2 a-1}+$ $k_{N, 2 a}=k_{N-1, a}$, this easily implies that $\sum_{a=1}^{2^{N}}\left|k_{N, a}\right|$ is constant for large $N$. In view of (2.19) and the compactness of the sets $K_{s}$, this proves that $\beta \in G$.

Now, let $\alpha_{1}, \alpha_{2}, \ldots, \alpha_{t}$ be given distinct elements of $K$. Then it is clear that if $N$ is large enough $\left(N \geq N_{0}\right)$, then for any $1 \leq a \leq 2^{N-1}$ we can take a $\delta(N, a) \in\{0,1\}$ such that

$$
\alpha_{1}, \alpha_{2}, \ldots, \alpha_{t} \notin K_{N, 2 a-\delta(N, a)},
$$

i.e.

$$
f_{N, 2 a-\delta(N, a), r}\left(\alpha_{i}\right)=0
$$

for every $r \geq 1,1 \leq i \leq t$. Then, defining $\delta(N, a) \in\{0,1\}$ arbitrarily for $0 \leq N<N_{0}, 1 \leq a \leq 2^{\bar{N}-1}$, by (2.5) and (2.36) we have

$$
\sum_{N=0}^{\infty} \sum_{a=1}^{2^{N-1}} \sum_{1 \leq r_{1}, r_{2} \leq R(N)+2} l^{1+\epsilon}\left(\left\|m_{j(N)}\left(f_{N, 2 a-\delta(N, a), r_{2-\delta(N, a)}}\right) \alpha_{i}\right\|\right)<\infty
$$

for $1 \leq i \leq t$. This, together with (2.14), means that defining $f$ on $A^{\star}$ by

$$
f\left(\left(m_{j(N)}\left(f_{N, 2 a-1, r_{1}}\right), m_{j(N)}\left(f_{N, 2 a, r_{2}}\right)\right)\right)=m_{j(N)}\left(f_{\left.N, 2 a-\delta(N, a), r_{2-\delta(N, a)}\right)},\right.
$$


(the definition is correct by (2.37)), and extending $f$ to $A$ by $f((n, n))=n$ for $n \in H$, we have (1.3) for every $1 \leq i \leq t$. We proved the existence of such an $f$ for $\alpha_{1}, \alpha_{2}, \ldots, \alpha_{t} \in K$, but since $K$ generates $G$, such an $f$ exists also for $\alpha_{1}, \alpha_{2}, \ldots, \alpha_{t} \in G$, in view of Lemma 2 (i). Then (1.1) follows easily, so the theorem is proved.

\section{Some lemmas}

Lemma 2. (i) There is a constant $M>0$ such that if $x, y \geq 0$, then

$$
l^{1+\epsilon}(x+y) \leq M\left(l^{1+\epsilon}(x)+l^{1+\epsilon}(y)\right) .
$$

(ii) There is an $m>0$ constant such that for any $a>0$ we have

$$
\sum_{r=0}^{\infty} l^{1+\epsilon}\left(2^{-r-a}\right) \leq m a^{-\epsilon} .
$$

Proof. For statement (i) we may obviously assume that $0<x, y<1 / 4$. Then

and so

$$
x+y \leq 2 \max (x, y) \leq \sqrt{\max (x, y)},
$$

$$
\begin{aligned}
l^{1+\epsilon}(x+y) \leq l^{1+\epsilon}(\sqrt{\max (x, y)}) & =\left(-\log _{2}(\sqrt{\max (x, y)})\right)^{-(1+\epsilon)} \\
& =2^{1+\epsilon} l^{1+\epsilon}(\max (x, y)),
\end{aligned}
$$

which proves (i). Statement (ii) is trivial from the definitions.

Lemma 3. If $\omega \in T, k \geq 1$ is an integer, and

$$
\|\omega\|,\|2 \omega\|,\|4 \omega\|, \ldots,\left\|2^{k} \omega\right\| \leq \delta<\frac{1}{10},
$$

then $\|\omega\| \leq \frac{\delta}{2^{k}}$.

Proof. This is easy, and proved as Lemma 3 of [B-S].

Lemma 4. If $\phi: T^{(2)} \rightarrow T$ is a group homomorphism and

$$
\limsup _{r \rightarrow \infty}\left\|\phi\left(\frac{1}{2^{r}}\right)\right\|<\frac{1}{4},
$$

then there is a unique integer $k$ such that $\phi(\alpha)=k \alpha$ for every $\alpha \in T^{(2)}$.

Proof. The uniqueness is obvious, we prove the existence. It is well-known that the Pontriagin dual of the discrete group $T^{(2)}$ is the additive group $\mathbf{Z}_{\mathbf{2}}$ of 2-adic integers. Hence there is a $0-1$ sequence $b_{r}(r \geq 0)$ such that

$$
\phi(\alpha)=\left(\sum_{r=0}^{\infty} b_{r} 2^{r}\right) \alpha
$$


for every $\alpha \in T^{(2)}$, hence

$$
\phi\left(\frac{1}{2^{r}}\right)=\frac{b_{0}}{2^{r}}+\frac{b_{1}}{2^{r-1}}+\ldots+\frac{b_{r-1}}{2}
$$

for every $r \geq 1$. We see from (3.3) that if $b_{r-1}=1, b_{r-2}=0$, then

$$
\frac{1}{2} \leq\left\{\phi\left(\frac{1}{2^{r}}\right)\right\} \leq \frac{3}{4}
$$

which is impossible for large enough $r$, in view of (3.1). Consequently, the sequence $b_{r}$ is constant for large enough $r$. If this constant is 0 , i.e. $b_{r}=0$ for $r \geq r_{0}$, then using (3.2), we get the lemma at once. If the constant is 1 , so $b_{r}=1$ for $r \geq r_{0}$, then, since

$$
\sum_{r=0}^{\infty} 2^{r}=-1
$$

in $\mathbf{Z}_{\mathbf{2}}$, one obtains the lemma from (3.2) with

$$
k=-1-\left(\left(1-b_{0}\right)+2\left(1-b_{1}\right)+\ldots+2^{r_{0}-1}\left(1-b_{r_{0}-1}\right)\right) .
$$

\section{Proof of Theorem 2}

If $G$ is a group and $d$ is a metric on $G$, we say that $(G, d)$ is a Polish group, if $d$ is a complete metric, and $G$ with this metric is a separable topological group.

The following proposition essentially appears on p. 541. of $[\mathrm{A}-\mathrm{N}]$, but since they give only a brief indication of the proof, we think that it is worth to include a proof here.

Proposition 1. Assume that $K$ is an uncountable compact subset of $T$, and $K$ is independent over $\mathbf{Z}$. Let $G \leq T$ be the subgroup generated by $K$. Let $d$ be a metric defined on $G$ such that $(G, d)$ is a Polish group. Then the injection map

$$
i:(G, d) \rightarrow T, \quad i(g)=g \text { for every } g \in G
$$

is not continuous (we take on $T$ its usual topology, inherited from $\mathbf{R}$ ).

Proof. Let $Q$ be a countable dense subgroup in $(G, d)$ (such a subgroup clearly exists, since $(G, d)$ is separable). Consider $Q$ with the discrete topology (discrete metric). Then $(Q, G)$ is a Polish (polonais) transformation group in the sense of $[\mathrm{E}]$, moreover, it clearly satisfies Condition $\mathrm{C}$ on $\mathrm{p}$. 41. of [E]. Since $Q$ is not locally closed in $G$ by our conditions, conditon (5) of Theorem 2.6 of $[\mathrm{E}]$ is not satisfied. Hence (9) of that theorem is also false, therefore there is a Borel measure $\mu$ on $G$ with $\mu(G)=1$ such that

(i) each $Q$-invariant measurable subset of $G$ has measure 0 or 1 ; 
(ii) each point of $G$ has measure 0 .

Indeed, $\mu(G)=1$ can be assumed, since $\mu$ is nontrivial and finite by [E], (i) follows since $\mu$ is ergodic in the sense of [E], and (ii) is true by (i), because $\mu$ is not concentrated in a $Q$-orbit.

The measure $\mu$ then has the following additional property, which is a strengthening of (ii):

(iii) if $F \subseteq G$ is a closed subset (in the $d$-topology) and $\mu(F)>0$, then there is an $A \subseteq F$ with $0<\mu(A)<\mu(F)$.

It follows by another application of Theorem 2.6 of [E]. Indeed, let $\{0\}$ be the trivial group, then $(\{0\}, F)$ is a polonais transformation group satisfying Conditon C on p.41. of [E], (5) of Theorem 2.6 is true, hence (8) of Theorem 2.6, using (ii), gives (iii).

Now, we are able to prove the proposition. Assume that $i:(G, d) \rightarrow T$ is continuous, and we will get a contradiction. For $t \geq 1, n_{1}, n_{2}, \ldots, n_{t} \in \mathbf{Z}$ set

$$
E\left(n_{1}, n_{2}, \ldots, n_{t}\right)=\left\{n_{1} x_{1}+n_{2} x_{2}+\ldots+n_{t} x_{t}: x_{1}, x_{2}, \ldots, x_{t} \in K\right\} .
$$

Every $E\left(n_{1}, n_{2}, \ldots, n_{t}\right)$ is a closed set in $(G, d)$, since it is closed in $T$ and $i$ is continuous. Since

$$
G=\bigcup_{t \geq 1} \bigcup_{n_{1}, n_{2}, \ldots, n_{t} \in \mathbf{Z}} E\left(n_{1}, n_{2}, \ldots, n_{t}\right),
$$

hence $\mu\left(E\left(n_{1}, n_{2}, \ldots, n_{t}\right)\right)>0$ for some values of the parameters.

Let $g \in G, t \geq 1, n_{1}, n_{2}, \ldots, n_{t} \in \mathbf{Z}$ be minimal with the property that

$$
\mu\left(g+E\left(n_{1}, n_{2}, \ldots, n_{t}\right)\right)>0,
$$

in the sense that

$$
\mu\left(h+E\left(m_{1}, m_{2}, \ldots, m_{r}\right)\right)=0
$$

for every $h \in G, r \geq 1, m_{1}, m_{2}, \ldots, m_{r} \in \mathbf{Z}$ with

$$
\left|m_{1}\right|+\left|m_{2}\right|+\ldots+\left|m_{r}\right|+|r|<\left|n_{1}\right|+\left|n_{2}\right|+\ldots+\left|n_{t}\right|+|t| .
$$

By (iii), writing $F=g+E\left(n_{1}, n_{2}, \ldots, n_{t}\right)$, there is an $A \subseteq F$ with $0<$ $\mu(A)<\mu(F)$. Then $\mu\left(\bigcup_{q \in Q}(q+A)\right)>0$, hence $\mu\left(\bigcup_{q \in Q}(q+A)\right)=1$ by (i). We prove that

$$
\mu\left(\left(\bigcup_{q \in Q}(q+A)\right) \bigcap(F \backslash A)\right)=0 .
$$

This will give a contradiction, because $\mu(F \backslash A)>0$. Since $Q$ is countable, it is enough to prove that $\mu((q+A) \bigcap F)=0$ for every $0 \neq q \in Q$, which follows, if we prove

$$
\mu((q+F) \bigcap F)=0
$$

for every $0 \neq q \in Q$. 
Assume that $q+f_{1}=f_{2}, f_{1}=g+e_{1}, f_{2}=g+e_{2}$, where $f_{1}, f_{2} \in F$, $e_{1}, e_{2} \in E\left(n_{1}, n_{2}, \ldots, n_{t}\right)$. For $i=1,2$ let

$$
e_{i}=n_{1} x_{i 1}+n_{2} x_{i 2}+\ldots n_{t} x_{i t}
$$

with $x_{i j} \in K$ for $i=1,2,1 \leq j \leq t$. Let

$$
q=\nu_{1} x_{01}+\nu_{2} x_{02}+\ldots \nu_{s} x_{0 s}
$$

with $s \geq 1$, and $\nu_{l} \in \mathbf{Z}, x_{0 l} \in K$ for $1 \leq l \leq s$. Since $q+e_{1}=e_{2}, q \neq 0$, and $K$ is independent over $\mathbf{Z}$, there are integers $1 \leq i \leq 2,1 \leq j \leq t$ and $1 \leq l \leq s$ such that $x_{i j}=x_{0 l}$. Therefore, if

$$
E:=\bigcup_{1 \leq l \leq s} \bigcup_{m \in \mathbf{Z}} \bigcup_{\left(r, m_{1}, m_{2}, \ldots, m_{r}\right) \in H}\left(m x_{0 l}+E\left(m_{1}, m_{2}, \ldots, m_{r}\right)\right)
$$

where

$$
H:=\left\{\left(r, m_{1}, m_{2}, \ldots, m_{r}\right): r \geq 1, m_{1}, m_{2}, \ldots, m_{r} \in \mathbf{Z},(4.2) \text { is true }\right\},
$$

then $e_{i} \in E$ for some $1 \leq i \leq 2$. Hence

$$
f_{2} \in(g+E) \bigcup(g+q+E) .
$$

Since $\mu(g+E)=\mu(g+q+E)=0$ by (4.1), (4.2), so (4.3) is true, and the proposition is proved.

Proof of Theorem 2. Assume that

$$
G=\left\{\beta \in T: \lim _{n \in A}\|n \beta\|=0\right\}
$$

for some infinite $A \subseteq \mathbf{Z}$. For $x, y \in G$ let

$$
d(x, y)=\|x-y\|+\max _{n \in A}\|n(x-y)\| .
$$

It is clear that $d$ is a metric on $G$, and $(G, d)$ is a topological group. We show that $d$ is complete. Let $\beta_{j} \in G, j \geq 1$ be a Cauchy sequence with respect to $d$. Then $\beta_{j}$ is a Cauchy sequence also in $T$ by (4.4), so there is a $\beta \in T$ such that $\left\|\beta_{j}-\beta\right\| \rightarrow 0$ as $j \rightarrow \infty$. Now, for $n \in A, j_{1}, j_{2} \geq 1$ we have

$$
\left\|n\left(\beta_{j_{1}}-\beta\right)\right\| \leq\left\|n\left(\beta_{j_{1}}-\beta_{j_{2}}\right)\right\|+\left\|n\left(\beta_{j_{2}}-\beta\right)\right\| .
$$

Letting $j_{2} \rightarrow \infty$ for fixed $n$ and $j_{1}$ we get

$$
\|n \beta\| \leq\left\|n \beta_{j_{1}}\right\|+\limsup _{j_{2} \rightarrow \infty} d\left(\beta_{j_{1}}, \beta_{j_{2}}\right),
$$

and $\beta_{j_{1}} \in G$ gives

$$
\limsup _{n \in A}\|n \beta\| \leq \limsup _{j_{2} \rightarrow \infty} d\left(\beta_{j_{1}}, \beta_{j_{2}}\right)
$$


for every $j_{1} \geq 1$, which proves $\beta \in G$. Let $\epsilon>0$, then we can take $j_{2}, N \geq 1$ so that

$$
\left\|n\left(\beta_{j_{2}}-\beta\right)\right\|+\sup _{j_{1} \geq j_{2}} d\left(\beta_{j_{1}}, \beta_{j_{2}}\right)<\epsilon
$$

for every $n \in A,|n| \geq N$. Hence for $j_{1} \geq j_{2}, n \in A,|n| \geq N$ we have $\left\|n\left(\beta_{j_{1}}-\beta\right)\right\|<\epsilon$ by (4.5). Since for any fixed $|n|<N$ we know that $\left\|n\left(\beta_{j_{1}}-\beta\right)\right\| \rightarrow 0$ as $j_{1} \rightarrow \infty$, this proves $d\left(\beta_{j_{1}}, \beta\right) \rightarrow 0$, so $d$ is complete.

Let $X$ be a countable dense subset in $T$, and for $N, l \geq 1$ integers, $x \in X$ let

$$
U_{N, l, x}=\left\{\beta \in G: \begin{array}{rl}
\|\beta-x\| & +\max _{n \in A,|n|} \leq N\|n(\beta-x)\| \\
& +\max _{n \in A,|n|}>N\|n \beta\|<\frac{1}{l}
\end{array}\right\} .
$$

It is easy to check that if we take an element from each nonempty $U_{N, l, x}$, then we get a countable dense subset of $(G, d)$. So the conditions of Proposition 1 are satisfied, hence $i:(G, d) \rightarrow T$ is not continuous. But this contradicts (4.4), so the theorem is proved.

\section{Some remarks and problems}

If $K$ is finite, it follows from [Bi2], Theorem 1 (ii) that Theorem 1 of the present paper would be false for $\epsilon=0$. But we cannot decide the following

Problem 1. Let $K$ be uncountable. Is Theorem 1 true with $\epsilon=0$ ?

The following proposition is a consequence of [V], p.140, Theorem 2' (the quoted theorem of Varopoulos is stronger than this statement):

Proposition 2. Let $L \subseteq T$ be a compact set with $L \cap G=\emptyset$, then there is an infinite subset $A \subseteq \mathbf{Z}$ such that

$$
G=\left\{\beta \in G \cup L: \lim _{n \in A}\|n \beta\|=0\right\} .
$$

Compare Proposition 2 with our Theorem 2. We do not know whether Proposition 2 can be strengthened in the following way:

Problem 2. Let $L \subseteq T$ be a compact set with $L \cap G=\emptyset$. Is there an infinite subset $A \subseteq \mathbf{Z}$ such that

$$
G=\left\{\beta \in G \cup L: \lim _{n \in A}\|n \beta\|=0\right\},
$$

and

$$
\sum_{n \in A}\|n \alpha\|<\infty
$$

for every $\alpha \in G$ ?

We state without proof our following partial result in this direction. 
Theorem 3. Let $L \subseteq T$ be a compact set with $L \cap G=\emptyset$, and let $v$ be a strictly increasing continuous function on the interva $[0,1 / 2]$ with $v(0)=0$. Then there is an infinite subset $A \subseteq \mathbf{Z}$ such that we have

$$
\sum_{n \in A} l^{1+\epsilon}(\|n \alpha\|)<\infty
$$

for every $\alpha \in G$, but

$$
\sum_{n \in A} v(\|n \beta\|)=\infty
$$

for every $\beta \in L$.

Remark that this theorem implies at once the result mentioned on p.40. of [H-M-P], namely that $G$ is a saturated subgroup of $T$ (for the definition of a saturated subgroup, see $[\mathrm{H}-\mathrm{M}-\mathrm{P}]$ or $[\mathrm{N}], \mathrm{Ch}$. 14). We note that the above-mentioned Theorem 2' on [V], p.140, also implies that $G$ is saturated.

Finally, we mention that Theorem 2 and Proposition 2 together show that if $K$ is uncountable, then $G$ is a g-closed but not basic g-closed subgroup of $T$ in the terminology of [D-M-T]. This answers the question of D. Dikranjan (oral communication) about the existence of such subgroups of $T$.

\section{References}

[A-N] J. AARONSON, M. NADKARNI, $L_{\infty}$ eigenvalues and $L_{2}$ spectra of no-singular transformations. Proc. London Math. Soc. (3) 55 (1987), 538-570.

[B] M. Beiglbock, Strong characterizing sequences of countable groups. Preprint, 2003

[Bi1] A. BIRó, Characterizing sets for subgroups of compact groups I.: a special case. Preprint, 2004

[Bi2] A. BiRÓ, Characterizing sets for subgroups of compact groups II.: the general case. Preprint, 2004

[B-D-S] A. Biró, J-M. Deshouillers, V.T. Sós, Good approximation and characterization of subgroups of $R / Z$. Studia Sci. Math. Hung. 38 (2001), 97-113.

[B-S] A. Biró, V.T. Sós, Strong characterizing sequences in simultaneous diophantine approximation. J. of Number Theory 99 (2003), 405-414.

[B-S-W] M. Beiglbock, C. Steineder, R. Winkler, Sequences and filters of characters characterizing subgroups of compact abelian groups. Preprint, 2004

[D-K] D. Dikranjan, K. Kunen, Characterizing Subgroups of Compact Abelian Groups. Preprint 2004

[D-M-T] D. Dikranjan, C. Milan, A. Tonolo, A characterization of the maximally almost periodic Abelian groups. J. Pure Appl. Alg., to appear.

[E] E. Effros, Transformation groups and $C^{*}$-algebras. Ann. of Math. 81 (1965), 38-55.

[H-M-P] B. Host, J.-F. Mela, F. Parreau, Non singular transformations and spectral analysis of measures. Bull. Soc. Math. France 119 (1991), 33-90.

[L-P] L. Lindahl, F. Poulsen, Thin sets in harmonic analysis. Marcel Dekker, 1971

[N] M.G. NADKARni, Spectral Theory of Dynamical Systems. Birkhauser, 1998

[V] N.Th. Varopoulos, Groups of continuous functions in harmonic analysis. Acta Math. 125 (1970), 109-154. 
András Biró

A. Rényi Institute of Mathematics

Hungarian Academy of Sciences

1053 Budapest, Reáltanoda u. 13-15., Hungary

E-mail : biroand@renyi.hu 